\title{
Automated Hand Hygiene Monitoring Systems: Current Issues, Developments and Perceived Benefits
}

\author{
Cawthorne $\mathrm{KR}^{1, *}$ and Cooke RPD ${ }^{1,2}$ \\ 'Department of Innovation, Alder Hey Children's NHS Foundation Trust, Liverpool, UK \\ ${ }^{2}$ Department of Medical Microbiology, Alder Hey Children's NHS Foundation Trust, Liverpool, UK
}

${ }^{\star}$ Corresponding author: K-R. Cawthorne, Department of Innovation, Alder Hey Children's NHS Foundation Trust, Liverpool, L14 5AB, UK; E-mail: katie-rose. cawthorne@alderhey.nhs.uk

Received: August 17, 2021; Accepted: August 23, 2021; Published: August 25, 2021

Healthcare-Associated Infections (HCAIs) are among the leading causes of death in the United States affecting about one in every 20 hospitalised patients [1]. Furthermore, at least $50 \%$ of these infections are preventable [2]. Healthcare Workers (HCWs) practicing good Hand Hygiene ( $\mathrm{HH})$ is fundamental to preventing HCAI transmission, as $\mathrm{HH}$ compliance and HCAI rates are closely linked [3]. Though this association is well established, maintaining high levels of $\mathrm{HH}$ compliance is a perennial problem in all healthcare settings. When independently audited, $\mathrm{HH}$ compliance has been shown to be in the region of $50 \%$ at best [4]. Compliance is particularly poor when staff are busy and experiencing burnout [5] which has been mirrored in a recent report that found hospital transmission of COVID-19 accounted for $20-25 \%$ of cases [6]. Stressed and overworked HCWs perform less $\mathrm{HH}$, thereby inadvertently increasing the risk of COVID-19 transmission and other HCAIs. Simply blaming HCWs is not the answer. Though maintaining adequate patient to staff ratios is essential, providing staff with unbiased and opportunistic feedback on their $\mathrm{HH}$ practice is also key to mitigating HCAI risk.

Accurately monitoring $\mathrm{HH}$ compliance is an important quality improvement and patient safety control strategy. Good $\mathrm{HH}$ compliance should be rewarded and celebrated. Equally, areas for improvement must be identified and educational resources allocated accordingly. The World Health Organisation (WHO) currently recommends that the gold standard for monitoring $\mathrm{HH}$ is by direct observation (DO) of HCWs using trained, independent auditors. In its technical manual, the WHO provides detailed guidance on the audit process stressing the value of immediate personalised feedback to HCWs [7]. Whilst DO is widely accessible and requires minimal financial investment, it is labour intensive and yet only produces small quantities of data [8]. A plethora of evidence demonstrates that the quality of DO data is impeded by the Hawthorne effect as well as observer and selection bias [9]. It has been estimated that the full hand hygiene audit cycle, including data input and feedback, can cost $£ 28,800$ per year in staff time using DO [10]. This could be considered a high price to pay for low quality data that ultimately gives Infection Prevention and Control (IPC) teams an unclear picture of the HCAI risk in their organisation. Whilst these issues are widely acknowledged in the IPC community, HCWs are also cognisant of the problems with DO. A survey conducted in 2020 found that $58 \%$ of 1,120 staff questioned did not strongly endorse $\mathrm{DO}$ as a method of monitoring $\mathrm{HH}$ [11]. "The Hawthorne Effect" was cited by HCWs as one of the reasons for not trusting $\mathrm{HH}$ data presented to them [11]. However, the same respondents were open to the introduction of innovative $\mathrm{HH}$ technologies. Though staff attitudes to new $\mathrm{HH}$ monitoring technologies have not always been reported to be so positive, other surveys have typically involved very small staff numbers and have not represented all staff groups $[12,13]$. Staff does appear to recognise that they change their $\mathrm{HH}$ behaviour when they are aware they are being audited by DO. Hence, if staff does not believe that HH compliance data reflects the real clinical environment they are working in, it then becomes easy to dismiss such skewed data and thus positive behavioural change is never achieved. Equally, getting front-line workers to buy-in to new approaches to HH monitoring, addressing their concerns about the accuracy of novel technologies and how this data will be used are also recognised as difficult challenges [14].

A number of automated hand hygiene monitoring systems (AHHMS) have been commercially developed to address these issues [15]. These systems use sensor technology to remotely monitor $\mathrm{HH}$ compliance, therefore reducing the need for human auditors, instead capturing large volumes of non-judgemental quantitative data. This benefit has already been realised by healthcare organisations that use AHHMS. One healthcare system was able to capture 35 million hand hygiene opportunities within the first six months of the COVID-19 pandemic [16]. The authors reported that their rich dataset allowed them to understand when changes in $\mathrm{HH}$ behaviour occurred and how long improvements in $\mathrm{HH}$ were sustained. Such robust data in similar quantities would not be achievable with DO alone, particularly when IPC staff resources were likely being diverted to COVID-19 containment measures.

There are three broad categories of AHHMS: group monitoring systems, badge-based systems, and video monitoring systems [8]. Group monitoring systems track usage of $\mathrm{HH}$ dispensers (soap and gel) to give an idea of $\mathrm{HH}$ events in a given location. Data produced from these systems can 'nudge' groups of staff to increase their usage of $\mathrm{HH}$ dispensers, but they cannot provide the personalised feedback that empowers HCWs to change their own practice [17]. Badge-based monitoring systems typically require HCWs to wear an additional 
tracking device that communicates with dispenser-based sensors. This extra layer of data is able to provide personalised feedback on $\mathrm{HH}$ behaviour both to the individual HCW, but also to managers and IPC teams. Implementing these types of AHHMS can be challenging because, due to their very nature, staff are required to wear an extra piece of equipment, which ultimately places an additional demand on staff to change their behaviour. In a study by Levin et al. [18], $44 \%$ of staff reported that wearing an additional tracking device was "inconvenient" to them. Both group monitoring and badge-based systems are usually unable to determine which WHO HH moment is being performed. They typically identify WHO moments one and four (before touching a patient and after touching a patient) [19]. This may be a useful surrogate in hospitals that have a high proportion of single rooms, however in many healthcare institutions (e.g. the National Health Service in the United Kingdom (UK)) this is not the case. Therefore, even where there is a successful implementation of an AHHMS, the continuous monitoring of $\mathrm{HH}$ events should be complemented by targeted DO to provide further qualitative insights into HCW HH behaviour; such as hand washing technique and types of $\mathrm{HH}$ opportunities missed.

Thirdly, video camera-based AHHMS can provide video footage that serves to replace human auditors. Video footage enables observation of all WHO five moments of hand hygiene and hand washing technique; however these systems have largely been confined to research rather than used commercially [20]. We expect this is due to patient privacy issues that may arise when video footage captures $\mathrm{HH}$ events in close proximity to patients. These privacy concerns could be alleviated through automated video auditing (AVA) which does not require storage or transfer of video data for analysis [21].

To our knowledge, there are currently 29 commercially available AHHMS, $75 \%$ of which are manufactured by companies based in the USA [15]. Over the last 10 years, uptake of these systems has remained stable, yet low, at around $4 \%[22,23]$. A small survey of Directors of Infection Prevention and Control (DIPCs) in the UK found that these systems were perceived to be expensive and not guaranteed to produce a return on investment [15]. In order for an IPC intervention to be considered cost-effective, it should reduce HCAI incidence by $15 \%$ [24]. Therefore, evidence is needed to demonstrate that AHHMS are able to reduce HCAI rates before these systems are likely to be adopted more widely. A recent survey on this issue found that only one AHHMS has randomised control trial (RCT) level evidence supporting its ability to reduce HCAI rates [15]. Here, a group monitoring AHHMS used a specialised stepped wedge cluster RCT (SWCRCT) study design to demonstrate a significant reduction in healthcare-associated methicillin-resistant Staphylococcus aureus rates when implemented as part of a multimodal IPC strategy [25]. Other infection types showed no significant change during the study. As this was a group-based monitoring system, it could be argued that improvements in HCAI rates were limited by the inability to provide personalised feedback to HCWs. Individualised feedback, whether given publicly or privately, has been repeatedly shown to improve $\mathrm{HH}$ compliance [26,27]. Whilst this particular SWCRCT was a promising start, more are needed where the AHHMS is a single intervention being investigated. This will hopefully provide the evidence needed to determine whether an AHHMS is likely to be a cost-effective method of driving down HCAI rates.

Alternatively, an AHHMS can be evaluated by assessing its impact on HH compliance as a primary end-point. A 2019 review of AHHMSs found that only one system has RCT-level evidence demonstrating its ability to increase $\mathrm{HH}$ compliance [28]. When this badge-based system was implemented it led to a small $6.8 \%$ increase in $\mathrm{HH}$ compliance [29]. Non-adherence to badge-wearing was, again, an issue in this study, with $21 \%$ of participants not wearing their device as required.

Whether improved HH compliance or reduced HCAI rates (or both) are the desired end-points for such systems, more RCT-level evidence is needed for each of the 29 systems currently available in the marketplace [30]. We expect that uptake of such systems will remain patchy until the evidence base improves.

In summary, the importance of both staff consultation on new approaches to improving $\mathrm{HH}$ compliance, and immediate personalised feedback to staff with individualized action planning cannot be overemphasized [27,31]. Furthermore, we would suggest that goal setting with reward incentives are incorporated into $\mathrm{HH}$ improvement strategies if they are to effect behavioural change [32]. AHHMSs are useful tools and well placed to achieve these aims as they can provide large volumes of quantitative data offering insights to IPC teams on HCW HH behaviour. Badge-based systems promise to deliver personalised feedback to staff on their performance, yet repeated studies have shown staff to be reluctant to wear said extra badges due to the inconvenience they cause. However, new developments in AHHMS need to ensure that they have no impact on staff workflow and that personalized staff feedback on $\mathrm{HH}$ performance becomes the norm. In addition, more RCT-level studies are required to demonstrate the efficacy of individual AHHMSs in reducing HCAIs. This will allow IPC professionals to make informed, evidence-based procurement decisions on whether a system is likely to be cost-effective for their organisation. Overall, there should be optimism about new developments in AHHMSs provided these can be aligned with an improved research and development supporting programme.

\section{References}

1. US Department of Health and Human Services (2013). National action plan to combat healthcare acquired infections-a road to elimination. Chapter 8: Long-Term Care facilities.

2. Zimlichman E, Henderson D, Tamir O, Franz C, Song P, et al. (2013). Health careassociated infections. A meta-analysis of costs and financial impact on the US healthcare system. JAMA Internal Medicine 173: 2039-2046. [crossref]

3. Sickbert-Bennett EE, DiBiase LM, Willis TMS, Wolak ES, Weber DJ, et al. (2016) Reduction of Healthcare-Associated Infections by Exceeding High Compliance with Hand Hygiene Practices. Emerging Infectious Diseases 22: 1628-1630. [crossref]

4. Pan, SC, Tien, KL, Hung, IC, Lin, YJ, Sheng WH, et al. (2013) Compliance of Healthcare Workers with Hand Hygiene Practices: Independent Advantages of Overt and Covert Observers. PLoS ONE 8: 53746. [crossref]

5. Manomenidis G, Panagopoulou E, Montgomery A. (2017) Job Burnout Reduces Hand Hygiene Compliance Among Nursing Staff. Journal of Patient Safety 15: 70-73. [crossref]

6. Scientific Advisory Group for Emergencies (2021) PHE and LSHTM: The contribution of nosocomial infections to the first wave. 
7. World Health Organisation. (2009) Hand Hygiene Reference Technical Manual. Geneva, Switzerland: World Health Organisation.

8. Boyce JM. (2019) Current issues in hand hygiene. American Journal of Infection Control 47: 46-52. [crossref]

9. Jeanes A, Coen PG, Gould D, Drey NS (2019) Validity of hand hygiene compliance measurement by observation: A systematic review. American Journal of Infection Control 47: 313-322. [crossref]

10. Jeanes A, Coen PG, Wilson AP, Drey NS, Gould DJ. (2015) Collecting the data but missing the point: validity of hand hygiene audit data. Journal of Hospital Infection 90: 156-162. [crossref]

11. Cawthorne KR, Cooke RPD. (2020) Healthcare workers' attitudes to how hand hygiene performance is currently monitored and assessed. Journal of Hospital Infection 105: 705-709. [crossref]

12. Blomgren P-O, Lytsy B, Hjelm K, Swenne CL. (2021) Healthcare workers' perceptions and acceptance of an electronic reminder system for hand hygiene. Journal of Hospital Infection 108: 197-204. [crossref]

13. Tarantini C, Brouqui P, Wilson R, Griffiths K, Patouraux P, et al. (2019) Healthcare workers' attitudes towards hand -hygiene monitoring technology. Journal of Hospital Infection 102: 413-418.

14. Conway LJ. (2016) Challenges in implementing electronic hand hygiene monitoring systems. American Journal of Infection Control 44: 7-12. [crossref]

15. Cawthorne K-R, Cooke RPD. (2021) A survey of commercially available electronic hand hygiene monitoring systems and their impact on reducing healthcare-associated infections. Journal of Hospital Infection 111:40-6. [crossref]

16. Moore LD, Robbins G, Quinn J, Arbogast JW. (2021) The impact of COVID-19 pandemic on hand hygiene performance in hospitals. American Journal of Infection Control 49(1):30-3. [crossref]

17. Hysong SJ, Best RG., Pugh JA. (2006) Audit and feedback and clinical practice guideline adherence: Making feedback actionable. Implementation Science 1(1). [crossref]

18. Levin PD, Razon R, Schwartz C, Avidan A, Sprung CL, et al. (2019) Obstacles to the successful introduction of an electronic hand hygiene monitoring system, a cohort observational study. Antimicrobial Resistance \& Infection Control 8(1).

19. Cheng VCC, Tai JW, Ho SK, Chan JFW, Hung KN et al. (2011) Introduction of an electronic monitoring system for monitoring compliance with Moments 1 and 4 of the WHO "My 5 Moments for Hand Hygiene" methodology. BMC Infectious Diseases 11.

20. Mckay KJ, Shaban RZ, Ferguson P. (2020) Hand hygiene compliance monitoring: Do video-based technologies offer opportunities for the future? Infection, Disease bै Health 25(2):92-100. [crossref]
21. Lacey G, Zhou J, Li X, Craven C, Gush C. (2020) The impact of automatic video auditing with real-time feedback on the quality and quantity of handwash events in a hospital setting. American Journal of Infection Control 48: 162-166. [crossref]

22. Braun BI, Kusek L, Larson E. (2009) Measuring adherence to hand hygiene guidelines: A field survey for examples of effective practices. American Journal of Infection Control 37: 282-288. [crossref]

23. Durant DJ, Willis L, Duvall S. (2020) Adoption of electronic hand hygiene monitoring systems in New York state hospitals and the associated impact on hospital-acquired C. difficile infection rates. American Journal of Infection Control 48: 733-739. [crossref]

24. Guest JF, Keating T, Gould D, Wigglesworth N. (2019) Modelling the costs and consequences of reducing healthcare-associated infections by improving hand hygiene in an average hospital in England. BMJ Open 9: 029971.

25. Leis JA, Powis JE, McGeer A, Ricciuto DR, Agnihotri T et al. (2020) Introduction of Group Electronic Monitoring of Hand Hygiene on Inpatient Units: A Multicenter Cluster Randomized Quality Improvement Study. Clinical Infectious Diseases 71: 680685. [crossref]

26. Fish L, Bopp D, Gregory D, Kerley KD, Gakhar S et al. (2021) Hand hygiene feedback impacts compliance. American Journal of Infection Control 49: 907-911. [crossref]

27. Stone SP. (2018) Time to implement immediate personalised feedback and individualized action planning for hand hygiene. JAMA Network Open 1: 183422. [crossref]

28. Meng M, Sorber M, Herzog A, Igel C, Kugler C (2019) Technological innovations in infection control: A rapid review of the acceptance of behavior monitoring systems and their contribution to the improvement of hand hygiene. American Journal of Infection Control 47: 439-447. [crossref]

29. Fisher DA, Seetoh T, May-Lin HO, Viswanathan S, Toh Y, et al. (2013) Automated Measures of Hand Hygiene Compliance among Healthcare Workers Using Ultrasound: Validation and a Randomized Controlled Trial. Infection Control \& Hospital Epidemiology 34: 919-928. [crossref]

30. Cawthorne K-R, Cooke RPD. (2020) Are electronic hand hygiene monitoring systems cost-effective? Stepped wedge cluster randomized controlled trials are needed to assess their impact on reducing healthcare-associated infections. Journal of Hospital Infection 106: 200-201. [crossref]

31. Cooke RPD, Corke C. (2020) Staff surveys will unlock the key to better hand hygiene performance. Lancet Infectious Diseases 20: 167-168. [crossref]

32. Luangasanatip N, Hongsuwan $M$, Limmathurotsakul D, Lubell $Y$, Lee AS, et al. (2015) Comparative efficacy of interventions to promote hand hygiene in hospital: systematic review and network meta-analysis. BMJ 351: 3728

\section{Citation:}

Cawthorne KR, Cooke RPD (2021) Automated Hand Hygiene Monitoring Systems: Current Issues, Developments and Perceived Benefits. Microbiol Immunol Pathol Volume 2(1): 1-3. 